\title{
Ueber das unicorneale Tracheaten- und speciell das Arachnoideen- und Myriopoden-Auge.
}

\author{
Von \\ V. Graber. \\ Hierzu Tafel V, VI und VII und ein Holzschnitt.
}

Nächst J oh. Müller's, Leydig's und M. Schultze's grundlegenden Arbeiten bezeichnet das wohl allerseits mit Ungeduld erwartete ausführliche Prachtwerk von Prof. Grenacher hinsichtlich der Erkenntniss der Arthropodenaugen und zwar, das Entwicklungsgeschichtliche ausgenommen, nach den verschiedensten Richtungen hin, unstreitig einen der grössten Fortsebritte und die darin angeregten Fragen und angedeuteten Lücken werden zweifelsohne auch zu neuen Forschungen wirksame Anregung geben.

Dass Letzteres aber grade bei mir der Fall war, erklärt sich darans, dass ich mich bereits i. J. 1875 eingehender mit der feineren Anatomie der Arachnoideen und speciell auch der Scorpioniden-Augen beschäftigte und schon nach der ersten Durchsicht des Grenacher'schen Opus sofort erkannte, dass meine einschlägigen Zeichnungen von der Darstellung des genannten Forschers in einigen und zwar grade in sehr wesentlichen allgemeinen Verhältnissen abweichen.

Dies bewog mich den Gegenstand neuerdings sorgfältig durchzuprifen, wobei, des Vergleiches wegen, auch ein Paar Spinnen sowie die von Grenacher bekanntlich völlig unbericksichtigt gelassenen und thberhaupt sehr vernachlässigten Myriopoden zur Untersuchung herangezogen wurden.

Indem ich hinsichtlich der einschlägigen älteren Literatur auf das Grenacher'sche Werk verweise, beziehe ich mich ausschliesslich nur auf jene Arbeiten, die mit den hier zu behandeln- 
den allgemeineren Fragen in unmittelbarem Zusammenhang stehen*), und schicke, da es zu keiner besonderen Besprechung der Myriopoden-Augen kommt, noch voraus, dass letztere in den wesentlichsten Puncten mit den Ocellen der Arachnoideen und den sog. Stemma's der Hexapoden ibbereinstimmen.

\section{A eussere Cuticula, Cornea-Linse.}

Aus Grenacher's sowie aus unseren Abbildungen (Fig. 4 und 22) geht evident hervor, dass die theils nach Aussen theils nach Innen ausgebogenen Cornea-Lamellen (c-l, aCu) continuirliche Fortsetzungen der allgemeinen Cuticularstraten sind, die am eingeschnürten Rande der Linse meist zu dünnen Platten zusammenschwinden, während sie in der Mitte zu dicken Balken anschwellen. $\mathrm{Ob}$ gewisse Linsenlamellen und namentlich die untersten vielleicht ohne Zusammenhang mit denen der Umgebung entstehen, d. h. ob die Linse vielleicht nach Innen auch völlig selbständige Schichten ansetzt, bleibt noch festzustellen.

Bezüglich der Corneaschichtung bei Phalangium, von welcher man nach Grenacher (p. 41) wenigstens bei mässiger Vergrösserung "Nichts wahrnimmt", sei bemerkt, dass ich dieselbe (nach Kalilaugebehandlung) schon mit Zeiss C. sehr deutlich unterscheide. Eine auffallend grobe Schichtung fand ich (Fig. 21, 22) an der verkalten Cornealinse von Julus. Bei Vespa crabro erwähnt und zeichnet (Fig. 31) Grenacher zwischen den inneren Linsenschichten eine grössere Zahl von Spalträumen, die er für Kunstproducte (beruhend auf Gerinnung und Volumsveränderung) ansieht. Eine ähnliche Klüftung zeigen auch mehrere Linsenschnitte von Buthus (Fig. $13 \mathrm{~h}$ ) und der Umstand, dass die betreffende nahe der Oberfläche gelegene weite Höhle von senkrecht zur Oberfläche verlaufenden Plas mafäd en oder Zellfortsätzen durchzogen ist, welche mit denen der weiten Poren

*) 1) Fr. L eydig, Zum feineren Bau der Arthropoden. Müller's Archiv f. Anat. und Phys. 1855; 2) Lehrbuch d. Histologie 1857; 3) Das Auge der Gliederthiere, Tübingen 1864; 4) Tafeln zur vergl. Anatomie, Tübingen 1864; 5) $\mathrm{H}$. Grenacher, Untersuchungen über das Sehorgan der Arthropoden $4^{0} 188 \mathrm{~S}$. mit 11 Tafeln Göttingen 1879. 
identisch sind, zeigt zur Genüge, dass dieser Sprung factisch nur auf eine durch die Härtung erzeugte Zusammenziehung der inneren Linsenschichten zurïckzuführen ist. An den Mittel- und Seitenaugen von Scorpio eur. (Fig. 3 und 4) haben wir dagegen eine solche Kontinuitätstrennung zwischen den oberflächlichen und tiefern Schichten niemals bemerkt und müssen demnach auch Blanchard's 1) Distinction einer gesonderten Cornea and Linse (cristallin) als der Wirklichkeit widersprechend zurückweisen.

Die völlige Uebereinstimmung in der Structur der Linse mit jener der allgemeinen Cuticula zeigt sich aber ganz besonders in der Verbreitung der bekanntlich zuerst von Le ydig näher studirten resp. entdeckten feinen Porenkanäle. Während nämlich Grenacher, im Gegensatz zu den Leydig'schen Angaben, p. 41 sagt: „Porenkanäle, wie sie Leydig gesehen hat, sind mir (an der Linse) nie zu Gesichte gekommen", und demgemäss auch auf keiner seiner Abbildungen dergleichen angedeutet sind, fanden wir solche ohne Ausnahme bei allen darauf untersuchten Gliederthieren, bei den Arachnoideen- so gut wie bei den Myriopoden- und Insectenstemma's.

Bei Anwendung einer starken Immersion (Zeiss L.) und im polarisirten Licht (bei eingeschobener Glimmerplatte) zeigt ein zu diesem Zweck möglichst dünn herzustellender und in Kalilauge aufgequollener Linsen-Axialschnitt ein ganz analoges Bild wie eine quergestreifte Muskelfaser, indem die einzelnen Linsenlamellen gleich den Discs in kleine durch mehr oder weniger breite dunkle Zwischenlinien scharf von einander gesonderte starklichtbrechende Stäbchen oder Stücke zerfallen. Zu solchen Studien empfiehlt sich besonders das Integument resp. die Linse von Julus, da hier die senkrechte Streifung (Fig. 22) schon bei mittelstarker Vergrösserung hervortritt und der Zerfall der Cuticularlamellen in anscheinend prismatische Elemente ausserordentlich prägnant zu Tage tritt.

Bei der Einstellung auf die Linsenoberfläche sieht man hier auch, dass es sich um wirkliche durch die ganze Dicke der Cuticula sich erstreckende Poren und keineswegs bloss um schwächer

1) L'Organisation du regne animal (Arachnides) par Emile Blanchard, p. 53 und Fig. 2 pl. IV. 
lichtbrechende Streifen, resp. um den Ausdruck einer Faserstructur handelt, denn die freie Fläche zeigt dasselbe „punctirte und gestrichelte" Aussehen, wie man es an den meisten dickeren Cuticularmembranen zu finden pflegt und auf die Mündungen sehr feiner Poren zurückführt.

\section{Hypodermis, Glaskörper, Pigmentzellen.}

Von der Existenz eines besonderen bereits von Joh. Müller entdeckten, aber erst durch Grenacber zur klaren Anschaung gebrachten subcornealen und $v o n$ der Retina unabhïngigen Zellstratums und dessen durch die pigmentirten Rand- oder Iriszellen vermittelten continuirlichen Ueberganges in die allgemeine Hypodermis habe ich mich beim Scorpion bereits im Herbst 1875 uberzeugt (Fig. 3), der Sache aber gegenüber der Leydig'schen Darstellung desshalb kein Gewicht beigelegt, weil mir das Vorkommen einer eigenen Linsen-matrix als selbstverständlich erschien.

Dass übrigens Leydig selbst der richtigen Auffassung von der Zweischichtigkeit des ocularen Weichkörpers sehr nahe war, beweist einmal die auch von Gre na eher erwähnte Stelle desselben betreffs „der zelligen Zeichnung an der Innenfläche der Linse, bei der Maulwurfsgrille (3 pag. 36) und dann die andere, wo er beim gleichen Thier von einem ,,irisartigen Gürtel" spricht, der sich von der Matrix der Cuticula her gegen die Linse erstreckt.

Im Uebrigen habe ich hier zu den ausgezeichneten Darstellungen Grenachers nur wenig beizufügen. Da Grenacher die Grenzconturen der Glaskörperzellen meist mit einer einfachen und nur auf Fig. 13 mit einer Doppellinie bezeichnet, könnte es scheinen, als ob diese ,abgestutzt pyramidenförmigen" Elemente uiberall hart aneinander lägen. Dass dies nicht der Fall ist, zeigt aber das Flächenbild von Scolopendra (Fig. 19 A). Jede Zelle besitzt hier eine selbständige scharf doppeltkonturirte Wand und zwischen den einzelnen doppelramigen Feldern dieser Mosaik befinden sich $z$. Th. sehr beträchtliche Intercellularräume, die wohl nicht ganz auf eine Schrumpfung der Zellen durch die Härtung zurtuckzuführen sein möchten.

Bezüglich der Kerne der Glaskörperzellen fügen wir bei, dass sie (vgl. Fig. 14, 19 und 25) meist verhältnissmässig etwas 
grösser sind, als sie auf den Grenacher'schen Zeichnungen (z. B. Fig. 22) erscheinen, gleichfalls eine deutlich doppelconturige Wandschicht besitzen (Fig. 19) und in der Regel ein besonderes Kernkörperchen erkennen lassen. Ihr Aussehen im frischen Zustand zeigt Fig. 27, gl, a.

Hinsichtlich der sog. Pigmentzellen erwähne ich zunächst, dass dieselben, wie dies Grenacher fur gewisse Spinnen und Insecten angibt, auch an den Seitenaugen der Scorpione sowie bei den Myriopoden wenig entfaltet oder differenzirt sind. Einen sehr distincten Pigmentzellenring findet man dagegen an den Mittelaugen von Buthus (Fig. 13 Ir), und tritt diese Iris, namentlich bei der Flächenansicht des Auges von Innen und nach vorheriger behutsamer Ablösung des Retinapolsters als breites Diaphragma sehr schön hervor. An feinen Schnitten überzeugt man sich ferner, dass die Verdickung der hypodermalen Pigmentzone im Umfang der Linse keineswegs immer auf einer beträchtlichen Verlängerung der Randzellen, sondern vielmehr darauf beruht, dass letztere ihrer ganzen Länge nach, die Hypodermzellen der weiteren Umgebung aber nur am äusseren Ende pigmentirt sind. Wir haben also hier einen relativ einfachen, mehr auf die chemische als auf die.morphologische Beschaffenheit gerichteten Differenzirungszustand.

Interessant ist die hypodermale Pigmentzellenzone im vordern Mittel- oder Stirnauge von Epeira, auf die wir auch noch später zurtickkommen. Grenacher stellt die betreffenden Elemente (auf Fig. $18 \mathrm{~A}$ bei $\mathrm{GK}_{1}$ ) als geradläufige Fasern dar, die den Kern ziemlich in der Mitte tragen. Nach meinen Präparaten (Fig. $25 \mathrm{pz}$ ) sind dieselben etwas anders. Man beachte einmal, dass die inneren d. h. dem Glaskörper zunächst liegenden Sförmig und die mittleren schwach sichelförmig gekrummt sind. Auf diese Art wird es möglich, dass dieselben trotz des flügelartig nach Aussen erweiterten Raumes, in dem sie sich befinden, mit beiden Enden die zugehörige Cuticula rechtwinkelig schneiden, ein Princip, das bekanntlich in der Architektonik der Pflanzengewebe sehr allgemein herrseht. Aus der Zeichnung ersieht man ferner, dass, dem Ausbreitungsraum entsprechend, nur die äusseren resp. cornealen Enden dieser Zellen eine grössere Breite haben, während sie nach Innen, gegen die Retina, in zarte Fasern sich verschmächtigen. $\mathrm{Ob}$ die Kerne gerade in der Mitte liegen, konn- 
ten wir nicht konstatiren, wohl aber, dass sie in manchen entschieden peripherisch situirt sind.

Eine Gegenüberstellung von Fig. 14 und 25 zeigt, dass beim Scorpion die hypodermalen Randzellen gerade die entgengesetzte Lage einnehmen.

Hypodermale Grenzhaut und Augenhülle.

Wie u. A. auch von uns seinerzeit klargestellt wurde ${ }^{1}$ ) entsteht theils durch Verschmelzung der Fussplatten der Hypodermiszellen, theils auch durch besondere Ausscheidung eine zusammenhängende innere (relativ zarte) Cuticula, die aber wohl zu unterscheiden ist ron gewissen echt bindegewebigen Basalmembranen, wie ich eine solche neuerlich wieder als separaten Beleg der inneren Cuticula an den Puppen und Imago's verschiedener Schmetterlinge beobachtet habe. (Vgl. auch den einschlägigen Aufsatz in diesem Archiv Bd. X. Fig. 10).

Eine solche echt-cuticulare Grenzlage findet sich nun auch, wie es scheint, ganz allgemein am Integument der in Rede stehenden Thiere, und kann man sich, wie meines Wissens zuerst Leydig bei der Biene (4) erkannt, an dünnen Schnitten leicht überzeugen, dass diese Membran (Fig. 4 und $14 \mathrm{vgr}$ ) am Umfang: des Stemma continuirlich auf das letztere und, in weiterer Fortsetzung, in das Neurilemm des Sehnervs übergeht, und auf diese Art also die Hülle oder Sclera des Auges bildet. Diese retinale Cuticula zeichnet auch Grenacher auf den meisten seiner Figuren und erwähnt (p. 45) speciell bei Epeira ausdrticklich, „dass beide Augen von einer feinen Cuticula überzogen werden, die sich einerseits nach der Hypodermis, andererseits auf den n. opticus fortsetzt".

Wenn aber die Augenhülle wirklich eine Cuticula ist, so versteht es sich von selbst, dass auch eine besondere Matrix zugegen sein muss, welche sie absondert. Letzterer wird aber von Grenacher bei den Spinnen nirgends gedacht und geben auch die einschlägigen Zeichnungen keinerlei Andeutung derselben. Nur am Stemma von Vespa Crabro (Fig. 34) findet man sowohl am Neurilemm des

1) Die tympanalen Sinnesapparate der Orthopteren. Denkschr. d. Kais. Akademie d. Wissensch. Wien 1875 (vergl. insbes. Fig. 66). 
Sehnervs als auch an der Sclera zahlreiche kleine (durch Hämatoxylin blau gefărbte) Kerne, und im Text (p. 60) die Bemerkung „die feine Cuticula, welche Auge und Nerv einschliesst, ist nur in letzterer Figur (34) gezeichnet, zugleich mit den Kernen, welche zu ihr gehören", und die also wohl nur auf die SeleraMatrix bezogen werden können.

Ausgezeichnet schön fanden wir nun letztere speciell bei Scolopendra (Fig. 18) nach theilweiser Auflösung des blauvioletten peripherischen Augenpigmentes durch Kalilauge. Die Sclera (sc) ist hier dick uud deutlich geschichtet. Dárunter sieht man dann, am deutlichsten im Anfang der Kalieinwirkung, theils runde vorwiegend aber spindelförmige ziemlich grosse Kerne (k), die sich durch Aufspeicherung des gelösten Pigmentes intensiv blau färben. Da diese Kerne der Cuticula sich eng anschmiegen, so kann es an dickeren Schnitten den Anschein gewinnen, als ob sie, wie an einer echten bindegewebigen Membran, in der letzteren selbst eingebettet wären.

Wenn ubrigens Leydig (4) auf Tafel IX Figur 4 e die Augenhülle wirklich als eine bindegewebige Schicht darstellt, so scheint uns dies - abgesehen davon, dass die betreffende Strecke, wie später zu zeigen, dem Glaskörper entspricht - im Widerspruch zu stehen mit seiner so klaren und auch von uns bestätigten Darstellung auf anderen Figuren seines klassischen Tafelwerkes, wo, wenigstens an der Nervenscheide, tiberall eine deutliche aussere Cuticula und eine dieser innen anliegende meist pigmentirte Matrixschichte mit hellen Kernen gezeichnet ist.

Beziuglich der in Rede stehenden Grenzhaut mache ich noch speciell auf die Strecke derselben unterhalb der "Pigmentzellen" aufmerksam. Hier . stehen nämlich (Fig. 25 x) die zugehörigen Hypodermzellen z. Th. nicht senkrecht auf der genannten Haut, sondern laufen vielmehr wie man sieht, derselben parallel, und ihre Abscheidung muisste also, falls sie tuberhaupt eine Cuticula ist, von der Seite her erfolgen.

Praeretinale Zwischenlamelle.

So nenne ich die am Tracheatenstemma bisher völlig unbeachtet gebliebene cuticulare Membran (Fig. 14 la), welche sich als 
selbstständige, jedoch mit der parietalen Sclera sowie mit der allgemeinen Grenzhaut continuirlich verbundene Schichte zwischen Glaskörper und Retina einschiebt.

Anscheinend hat zwar dieses die zwei Hauptstraten des Augenweichkörpers scheidende Septum bereits Leydig beobachtet, es lässt sich indess leicht nachweisen, dass dies nicht der Fall ist. Der vorhin erwähnte „bindegewebige Saum" nämlich mit „den zahlreichen darunter liegenden runden Kernen“, welche Leydig speciell an der Horniss „namentlich am Vorderrand (des Auges) wahrgenommen" (3, pag. 33) und auf seiner IX. Tafel zur vgl. Anatomie (Fig. 4 e) gezeichnet hat, kann, wie auch Grenacher (p. 62) bemerkt, nur auf den sehr dünnen Glaskörper bezogen werden, während die wirkliche $Z$ wischenlamelle hier von kaum messbarer Dicke ist und keinerlei Kerne enthält.

Dass aber auch Grenacher diese Membran entgangen ist, beweist sehon die angezogene Stelle (p. 62), wo er Leydig's Ansicht, nach welcher sich die retinale Hüllmembran ,auch iber die Vorderfläche der Retina hinüberschlage", als ,irrthümlich"bezeichnet, sowie die Darstellung auf seinen Zeichnungen, auf die wir kurz eingehen muissen.

Während dieser Forscher bekanntlich die Retinaelemente im Larvenauge von Dytiscus u. A., nur als etwas aus der Reihe der Hypodermiszellen ausgetretene resp. nach Innen gerückte oder versenkte Gebilde hinstellt, macht er hinsichtlich des Spinnen- und des imaginalen Insectenstemmas schon in der Einleitung (p. 40) eigens darauf aufmerksam, dass hier die Retina ganz ,aus dem Verband (der Hypodermis) ausgeschieden ist" und sagt dann speciell bei Phalangium (p. 41) noch ausdrticklich: ,auf Schnitten kann man immer mit vollster Sicherheit die hintere Abgrenzung des Glaskörpers als eine sehr scharfe vor den Stäbchenenden gelegene Linie nachweisen."

Dass Grenacher aber unter dieser Grenzlinie keine eigene Membran versteht, ergibt sich zur Evidenz aus den einschlägigen Zeichnungen. Mustert man z. B. das Vorderauge von Epeira in seiner Fig. 18 A (rechts), so sieht man Folgendes. Unterhalb der bogenförmigen Reihe der Glaskörperkerne zieht eine dunkle e infache Linie (Vgl. auch s. Fig. 19 u. 20). Diese Linie bricht dann in der Nähe der Rand- oder Pigmentzellen plötzlich ab, und 
letztere selbst erscheinen unmittelbar den äussersten (parietalen) Elementen der Retina angelehnt.

Wäre das Lagerungsverhältniss nun wirklich so, wtirden sich also, wie insbesondere auch a uf seiner Fig. $31 \mathrm{Pg}$ (Vespa crabes) unzweideutig a usgedrückt ist, die Pigmentzellen unmittelbar und Glied für Glied an die Seiten der sog. Retinazellen anschliessen, so wäre ja thatsächlich ein continuirlicher Uebergang $v 0 n$ den letzteren zu den echten Hypodermiszellen vorbanden ') und könnte sonach auch das typische zweischichtige Stemma nur als eine Modification des anscheinend einschichtigen Dytiscus-Larvenauges betrachtet werden.

Eine solche directe Verbindung der Retina mit den das Auge umsäumenden Integumentzellen existirt aber nicht; Hypodermis, Pigment- und Krystallkörperzellen einerseits und Retina anderseits bilden vielmehr je ein geschlossenes Ganzes für sich, indem sich eben zwischen beiden Straten unser präretinales Septum durch und durch zieht, und so vielleicht auch für die Zulässigkeit der Grenacher'schen Theorie bezüglich des hypodermalen (wir sagen nicht ectodermatischen) Ursprungs der Arthropoden-Retina eine schwer zu uberwindendeSchranke bildet.

Das Weitere zeigt zunächst Fig. 25 (von Epeira) und deren Vergleichung mit Grenachers Fig. $18 \mathrm{~A} \mathrm{Gk}_{1}$. Unter dem eigentlichen Glaskörperstratum sieht man am betreffenden Präparat bei genügend starker -Vergrösserung nicht eine einfache Linie, sondern deutlich einen doppeltkonturirten Grenzsaum, ja, an hinlänglich dünnen Schnitten innerhalb desselben noch ein Paar distincte Linien als Ausdruck einer allen dickeren Cuticularhäuten zukommenden Schichtenbildung. Dieses Band (la) hört nun aber keineswegs, wie in Grenacher's Figur, an der Grenze der Pigmentzellen auf, sondern man sieht es deutlich in einem scharfen Bogen gegen die äussere Augenhillle hinziehen und dort (bei v) einerseits mit der letzteren (oc), andererseits mit der allgemeinen Innen-

1) Wenn Grenacher pag. 158 hinsichtlich des Spinnen- und InsectenStemma's sagt, „hier ist die Retina in den von mir untersuchten Zuständen ausser aller Continuität mit der Hypodermis", so steht diese Aeusserung entschieden im Widerspruch mit den erwähnten Zeichnungen. 
Cuticula der Hypodermis (gr) verschmelzen. Demgemäss betrachte ich die Zwischenlamelle als directe Fortsetzung der integumentalen Binnen-Cuticula, von der sich nach Innen, gegen den n. opticus, die eigentliche Sclera abzweigt.

Noch leichter nachzuweisen, weil beträchtlich dicker, ist diese wichtige Demarkationsmembran bei den Scorpionen. Dieselbe erreicht hier (Fig. 14 la) unter dem Glaskörper eine Mächtigkeit von $0,002 \mathrm{~mm}$ und ist deutlich geschichtet. Durch Maceration der Schnittpräparate gelang es hier auch dieselbe stellenweise zu isoliren und konnten wir uns so tiberzeugen, dass sie wirklich eine selbständige Gewebslage darstellt.

Wie ich schon an älteren Zeichnungen dargestellt finde, ist die Zwischenlamelle an ihrem Rande, d. i. dort, wo sie sich in den einerseits gegen die Haut und andererseits gegen die Retina ziehenden Schenkel spaltet, beträchtlich verdickt und fanden wir neuerlich eine solche ringförmige im Durchschnitt dreieckige Anschwellung auch bei Buthus (Fig. 14 v) ausgebildet. Hier erkennt man mit Immers. L. auch sehr schön die Lamellen, die von diesem Knotenpuncte aus nach drei verschiedenen Richtungen divergiren.

Indem ich noch beifüge, dass dasselbe Septum u. A. auch bei den Myriopoden ( $\nabla g$ l. Fig. 17 v) vorkommt, möchte es wohl angezeigt sein, die Larvenaugen von Dytiscus speciell nach dieser Richtung einer neuerlichen Prüfung zu unterziehen; a priori glaube ich, dass auch sie eine solche $\mathrm{m}$. limitans haben, und dass sich also Grenachers Angabe von der Einschichtigkeit ihres Weichkörpers nicht bewahrheiten wird ${ }^{1}$ ).

\section{Retina.}

Ganglienzellen- und "Stäbchenzellen"-Schichte.

Während die den Grena cher'schen Untersuchungen zunächst vorausgehenden Angaben von Leydig betreffs der von ihm angenommenen continuirlichen Verschmelzung der Stäbchen- mit den Krystallzellen in der That sich als unrichtig erweisen, verdienen, wie sich zeigen wird, dessen allerdings etwas spärliche Daten über

1) Neustens beobachtete ich ein solches Septum in der That. 
die ganglienzellenartigen Elemente des inneren Stratums mehr Beachtung, als ihnen Gren acher schenkt, welcher Letztere bekanntlich eine Lage besonderer intraoculärer Ganglienzellen ganz läugnet und ganz allgemein (p. 158) die gesammte Längsstreke der Netzhaut-Pallisaden vom Eintritt des Opticus in den Augenbecher bis zum Glaskörper als eine einzige and einkernige Zelle betrachtet.

Die erwähnte Darstellung L e ydig's speciell an der bekannten Fig. 135 seiner Histologie (p. 256) und zwar nach einem ,senkrechten (wahrscheinlich etwas dicken) Schnitte vom Salticus-Auge ist folgende. Der oculäre Weichkörper zeigt zwei Hauptschichten, eine ganz helle Aussenzone (e), der Glaskörper, und eine dunkel pigmentirte Innenzone (f), "die Stäbchenschioht." Jede dieser Zonen zeigt ferner hinten eine Lage grosser heller kernartiger Bläschen.

Berücksichtigt man nun zunächst hinsichtlich der äusseren Kernzone, dass die Nuclei der eigentlichen Stäbchenzellen, wie auch Grenacher bemerkt, oft nur sehr schwer zu sehen sind, meist kein distinctes Binnenkörperchen (wie an den Leydig'schen Vorderkernen ein solches gezeichnet ist) erkennen lassen, während andererseits die gerade in diese Zone fallenden Glaskörperkerne auch an minder gelungenen Präparaten auf den ersten Blick sich präsentiren, so erscheint es mir, gleich Grenacher, ganz zweifellos, dass diese Leydig'schen Vorderkerne in der That dem letztgenannten Hypodermalgewebe angehören.

Was nun aber die Kerne hinter den Stäbchen resp. am Grunde des Auges betrifft, so machen dieselben wegen ihrer Grösse und Deutlichkeit durchaus nicht den Eindruck jener meist unscheinbaren kleinen Nuclei, wie sie Grenacher auf seinen Stäbchenzellen zeichnet, während sie nach Lage und Form mit den von uns später zu besprechenden Ganglienzellen übereinstimmen, und da Leydig in der Erkenntniss der Nervenelemente gewiss sehr bewandert, (p. 253 seiner Histologie) noch ausdricklich bemerkt, dass in der Spinnen-Retina „bi polare Gangli enkugeln" existiren, so haben wir keinen Grund daran zu zweifeln, dass Leydig wirklich die Sonderung der Retinastrahlen in eine basale Nervenzelle und in ein terminales Stäbchen beobachtet hat, und wenn Grenacher (p. 56) bemerkt, "was Leydig als ganglienkugelartige Zelle bezeichnet, dürfte bei Lycosa wahr- 
scheinlich der vor dem Stäbchen gelegene kolben- und kernführende Theil der Retina sein, während bei dem von ihm abgebildeten Auge von Salticus die Kerne sicher den Glaakörperzellen angehören", so kann Letzteres doch nur für die früher besprochene Leydig's che Vorderkernzone unmöglich aber für die hintere Lage (g) Giltigkeit haben, da letztere auf der in Rede stehenden Figur ja thatsächlich hinter der Stäbchenschichte und überhaupt ganz rückwärts, im Grunde des Auges liegt.

Wir gehen nun auf Grenacher's Darstellung über, wobei hinsichtlich seiner Auffassung vom stemmalen Retinaelement vor Allem hervorzuheben ist, dass er in demselben, wie schon angedeutet, nur einen einzigen Kern auffand und allgemein annimmt, und demzufolge auch dasselbe als ein einfaches Zell-Indi$\nabla$ id u u betrachtet.

Aus der Vergleichung seiner einschlägigen zahlreichen Abbildungen ist aber zunächst leicht zu ersehen, dass der Kern seiner "Retinazelle" in Bezug auf dessen Lage und z. Th. auch betreffs seiner Beschaffenheit und des Verhaltens der zugehörigen Strecke des Retinastrahles bei den verschiedenen Thieren resp. bei den verschiedenen Augen eines und desselben Geschöpfes einen sehr ungleichen Werth besitzt.

Mit Rücksicht auf die Lage zunächst erscheint Grenacher's Retinalzellenkern bald als ein apikales d. i. an das äussere oder vordere Ende des Retinalschlauches geriucktes Gebilde, bald als ein mehr oder weniger basales, indem es weit nach hinten gegen die Opticusfaser verschoben erscheint, und wollen wir vorläufig dieses Lagerungsverhältniss der Kerne und zwar zunächst mit Bezug auf das Stäbchen als prä- und als postbacilläres bezeichnen.

Präbacillär erscheint der Kern nach Grenacher im Hinterauge von Epeira (Fig. 18 Bk'), im Hinterauge von Lycosa (Fig. 22 und $23 \mathrm{k}^{\prime}$ ), ferner bei Phryganea (Fig. 35) und, aber in höchst eigenthümlicher Weise translocirt, am äusseren Vorderrand(Fig. $25 \mathrm{k}$ ) und am Hinterauge (Fig. $27 \mathrm{k}$ ) von Salticus.

Postbacillär hingegen in allen übrigen beobachteten Fällen: bei Phalangium (Fig. 15), am Vorderauge von Epeira (Fig. 18 A), desgleichen von Lycosa (Fig. 22k), dann am inneren Vorderrandauge von Salticus (Fig. $28 \mathrm{k}$ ), und unter den Insecten bei Musca (Fig. 30), bei Vespa (Fig. 31) und Crabro cr. (Fig. 34). 
Erwägt man nun: 1) dass der verhältnissmässig so selten zur Beobachtung gelangte präbacilläre Kern nach Grenacher's eigenen Angaben selbst am grossen Hinterauge von Epeira sehr „vergänglich" und schwer nachweisbar ist, und 2) dass der mit wenigen Ausnahmen allgemein erkannte postbacilläre Nucleus sowohl im Text (p. 44) als z. Th. auch auf den Zeichnungen als "ziemlich gross" und deutlich angegeben wird, so erscheint schon von vornherein die Annahme, dass Grenacher die Vorderkerne, namentlich an den relativ kleinen Ocellen der Spinnen und besonders der Insecten $z$. Th. tibersehen hat, und dass also diese sowie die meisten anderen Stemmata zwei durch Lage und Beschaffenheit von einander verschiedene Kernzonen haben, viel wahrscheinlicher als die Ansicht, dass die Retinades einen Auges nur Vorder-, die des anderen nur Hinterkerne besitze.

In Grenacher's Darstellung finden sich aber anch einige weitere Anzeichen dafür, dass im Hinterkerne wirklich ein vom präbacillären Nucleus scharf zu unterscheidendes besonderes Element vorliegt, und zwar ein solehes, das in Verbindung mit dem dasselbe dumgrenzenden Abschnitt des Retinaschlauches genau dem entspricht, was bereits Leydig als bipolare Ganglienzelle gedeutet hat.

Wer in Grenacher's Fig. 15 (Phalangium) die „spindelförmigen (ineinander gekeilten) A uftreibungen“ (p. 41) der Sehschläuche am Grunde des Retinapolsters mit dem gerade an diese Stelle fallenden „deutlichen“ Kern völlig vorurtheilslos und unbekümmert darum, ob für den ubbrigen Endabschnitt bereits ein Kern nachgewiesen ist oder nicht, ansieht, wird sich, denken wir, wohl kaum der Vorstellung erwehren können, dass hier in der That eine besondere Schichte ganglienzellenartiger Elemente vorliegt, aus denen sich die eigentlichen Endschläuche erheben.

Ein ähnliches Bild zeigt sich dann noch in Grenach er's Fig. $18 \mathrm{~A} \mathrm{k}$ (Vorderauge von Epeira) und wird auch im Text (p. 44) erwähnt: „Dass die Retinazellen in ihrer hinteren ke ulenförmigen Anschwellung einen deutlichen ziemlich grossen Zellkern" tragen. Wenn im Anschluss an diese Stelle Grenacher noch besonders bemerkt, „ich habe mich gentigend, auch nach Untersuchung von Tinctionspräparaten (mit Hämatoxylin) von dieser Lagerung überzeugt, um dies mit all' der Sicherheit, 
die eine oft und unter verschiedenen Umständen wiederholte Beobachtung gewähren kann, behaupten zu können", so hat es, wie uibrigens sehr leicht zu konstatiren ist, mit dieser Angabe auch in der That seine volle Richtigkeit.

Ich wende mich nun zu den eigenen Untersuchungen. Macht man einen einigermassen diunnen Median- oder überhaupt Tiefenschnitt durch ein Mittelauge yon Buthus, so sieht man nach Entfernung des Retinapigmentes, und zwar schon mit schwächeren Linsen zwei Bogenreihen von Kernen: eine vordere, am Glaskörper (Fig. $13 \mathrm{gl}$ ) und eine hintere (gz), fast am Grunde des Augenpolsters. Letztere Kerne treten besonders scharf hervor, was leicht begreiflich, da sie erstens ungefähr zweimal so gross sind $(0,01 \mathrm{~mm})$ als die Glaskörperkerne $(0,006)$, und als sie zweitens ein stark lichtbrechendes fast öltropfenartiges Aussehen haben. Der Lage nach entsprechen die Kerne offenbar den postbacillaren Gebilden.

Auch am dünnsten Schnitt, den man herzustellen im Stande, liegen diese Kerne, im Gegensatz zu jenen des Glaskörpers, niemals in einer Reihe, sondern, wenigstens in der mittleren Zone zu 2-4 unregelmässig hintereinander, jedoch in der Regel durch beträchtliche und ziemlich gleich grosse Intervalle getrennt, ein Umstand, der schon im vorhinein weniger auf ein Epithel, als vielmehr auf eine Schichte von unregelmässig neben und übereinander postirten Ganglien- oder überhaupt Kugelzellen hindeutet. - Man sieht ferner, dass diese Kerne am Mantel der Retina sich auch weiter nach oben hin ausbreiten, aber so, dass sie in ihrer Gesammtheit ein gegen den Rand des Retinabechers sich verdünnendes schalenförmiges Stratum bilden, indem nach der bezeichneten Richtung hin die Zahl der hintereinanderstehenden Kerne stetig abnimmt.

Im Wesentlichen die gleiche Anordnung und relative Grösse zeigen die retinalen Hinterkerne auch im Mittel- und Seitenauge (Fig. 4 gz) von Scorpio, nur dass bei letzterem und bei nur mittelstarker Vergrösserung weniger die grob granulirten Kerne selbst, als ihre $(0,003 \mathrm{~mm})$ grossen homogenen Kernkörperchen scharf hervortreten.

Mustert man nun einen möglichst dünen und durch geringe Maceration sowie durch feine Glasnadeln hinten gelockerten Retinaschnitt und zwar von Buthus mit Zeiss Immers. L, so ergibt sich das mit möglichster Treue copirte Bild Fig. 14. Die ge- 
gewissen Hinterkerne (gk) zunächst erscheinen als grosse, und wie schon bei schwacher Vergrösserung, stark lichtbrechende bräunlichgelbe Kugeln, im Innern mit mehreren blassrosa schimmernden Gebilden, von denen es uns, da wir sie nicht im frischen Zustand sahen, ungewiss ist, ob sie als Nucleoli zu deuten sind.

Die Figur zeigt dann ferner, dass jeder dieser grossen Kerne einer sog. bipolaren Zelle (gz) angehört.

Der hintere oder centripetale Fortsatz dieser Zellen, der übrigens, da er selten in die axiale Schnittrichtung fällt, nur ausnahmsweise vollständig zur Beobachtung gelangt, ist viel schmäler als der obere und verschmächtigt sich bald zu einer ca. 0,003 mm dicken und, wie auch an Querschnitten zu sehen, anscheinend vollkommen homogenen Opticusfaser.

Der vordere oder centrifugale Fortsatz hingegen erhebt sich in axialer Richtung als schlauchförmiges Gebilde nach oben und erscheint vom Ursprung bis zum Ende fast von gleichem Kaliber, wobei man stets um den körnigen Inhalt eine deutliche, meist scharf doppeltconturirte Membran unterscheiden kann.

Fasst man dies Alles zusammen und berücksichtigt ausser der Lage und Form dieser Gebilde besonders die auffallende Grösse des Kernes, sowie die der Kernkörperchen, so dürfte die Berechtigung, dieselben unter den Begriff der Ganglienzellen zu bringen, wohl kaum bestritten werden können.

Wir legen aber das Hauptgewicht durchaus nicht auf die Benennung des genannten Abschnittes, sondern auf die an unsern Buthus-Präparaten wiederholt konstatirte Thatsache, dass jeder Retinalschlanch nicht bloss einen basalen, sondern wenigstens noch einen zweiten und zwar einen apikalen Kern hat.

Hinsichtlich dieser Vorderkerne sei zunächst vorausgeschickt, dass sie sowohl wegen ihrer Lage (sie sind gleichsam eingeklemmt zwischen Septum und Stäbchen) als wegen ihrer relativen Kleinheit und ihres geringen Lichtbrechungsvermögens factisch oft ausserordentlich schwer zu bemerken sind, und dass also das gänzliche Uebersehen derselben von Seite der bisherigen Untersucher sehr leicht $\mathrm{zu}$ erklären ist.

Trotz dieser Unscheinbarkeit und z. Th. wohl auch „Vergänglichkeit" der Präbacillär-Kerne, die ja auch Grenacher betont, glauben wir uns aber doch von ihrer Existenz hinlänglich überzeugt zu haben. 
Stellt man, aber an einem stark (mit Kalilauge) entfärbten und dann wieder etwas angesäuerten Präparat auf die Gegend des präretinalen Septums ein, so sieht man ganz knapp hinter demselben, jedoch noch innerhalb der Retinalschläuche selbst, von Stelle zu Stelle einen durch das gelöste Pigment etwas intensiver als die Umgebung roth gefär'bten kreisrunden Fleck, z. Th. (Fig. $14 \mathrm{ak}$ ) mit deutlich doppelt konturirter Wandschicht und bisweilen mit einem winzigen stark lichtbrechenden Binnenkörperchen. Der Durchmesser dieser Kerne ist nur wenig grösser als jener der Nueleoli im Basalkern und um ein Kleines geringer als jene der fast unmittelbar angrenzenden Glaskörperkerne. Eine Verwechselung mit den letzteren ist übrigens bei einiger Achtsamkeit auch deshalb ausgeschlossen, weil, wenigstens an den vorliegenden Präparaten von Buthus, bei unserer Behandlungsweise die Glaskörperkerne sich gar nicht oder kaum merklich mit dem Augenfarbstoff imbibiren ${ }^{1}$ ).

Zwei polare; d. i. an den entgegengesetzten Enden der Retinaschläuche liegende Kerne mit ganglienzellenartiger Auftreibung des Basaltheiles findet man aber keineswegs bloss bei den Scorpioniden, wir konstatiren dasselbe Verhalten zunächst auch an dem vorderen Mittelauge einer Epeira, dem Grenacher ausschliesslich nur basale Kerne zuschreibt.

In Fig. 25 beachte man vor Allem die grossen Ganglienzellen resp. die kolbenförmigen Basaltheile der Retinaschläuche ( $\mathrm{gz}, \mathrm{gz} \mathrm{z}_{1}$ ), die ganz wie beim Scorpion vom Augengrund sich auch längs des Mantels nach oben ausbreiten, und überall eng in einandergekeilt und deshalb z. Th. polyedrisch umgrenzt erscheinen. Während die Endschläuche selbst im Mittel nur bei $0,007 \mathrm{~mm}$ breit sind, erreichen die Anschwellungen eine Dicke von 0,01 mm und darüber. Der zugehörige runde Kern (gk) ist aber relativ beträchtlich kleiner als beim Scorpion, indem er ungefähr dasselbe Kaliber $(0,006 \mathrm{~mm})$ wie die Glaskörperkerne besitzt. Derselbe imbibirt sich meist mit dem Augenpigment, besitzt ferner eine deutliche Wandschicht, ist grob granulirt and wegen dieser Eigenschaften schon mit schwacher Vergrösserung sofort zu erkennen. In der Regel bemerkt man auch ein kleines helles Kernkörperchen. Dieselben Verhältnisse zeigt auch E. diadema.

1) Umgekehrt nehmen die hypodermalen sowie die extraoculären Zellkerne viel leichter künstliche Farbstoffe auf als die retinalen. 
Vergleicht man Grenacher's Abbildung (Fig. 18 A) desselben Objectes mit der Wirklichkeit, so kann man sich nicht des Eindruckes erwehren, dass sie betreffs der in Rede stehenden Verhältnisse doch etwas allzu schematisch gehalten ist. Insbesondere sind die Basaltheile seiner Schläuche viel zu schlank und auch die Kerne zu länglich gezeichnet und möchten wir auch bezweifeln, ob je an einem Schnitt die mittleren oder axialen Schläuche immer direct in der geraden Verlängerung der Opticusfasern liegen, da an unseren Präparaten neben den der Länge nach getroffenen Schläuchen stets auch Querschnitte von Ganglienzellen sowie von Opticusfaserbündeln (Fig. $25 \mathrm{fa}$ ) sichtbar sind.

Ich will gleich hier beifugen, dass im Ganzen dieselben Verhältnisse, wie wir sie für die Ganglienzellenschichte der Scorpione und gewisser Spinnen eben angegeben haben, auch bei den Myriopoden, speciell bei Scolopendra (Fig. 17, $18 \mathrm{gz}$ ), Lithobius (Fig. 24) und Julus (Fig. $22 \mathrm{gz}$ ) wiederkehren. Zweifelhaft blieb mir aber bei Scolopendra, ob die so auffallend grossen runden Basalkugeln (Fig. 18) mit ihrer scharf doppelt-konturigen Wand und dem kleinen Binnenkörperchen als Kerne oder als Querdurchschnitte durch die Ganglienzelle selbst zu betrachten sind; grosse Basalkerne besitzt auch Julus (Fig. $22 \mathrm{gz}$ ).

Zum vorderen Epeira-Auge zurickkehrend ist nun das Vorhandensein der von Grenacher hier gänzlich übersehenen Präbacillär-Kerne (Fig. $25 \mathrm{ak}$ ) zu betonen. Man hat sie genau an derselben Stelle wie bei Buthus zu suchen, ihre Auffindang erfordert aber noch feinere Schnitte, eine subtilere Behandlung und eine grössere Geduld und Achtsamkeit. Ich kann nur sagen, dass ich sie und zwar unter ganz analogen Verhältnissen wie dort, wirklich als distincte körnige Kreisflecke von $0,0045 \mathrm{~mm}$, die der ganzen Erscheinung nach an die gleichliegenden und also anch gleichwerthigen Nuclei am Hinterauge (Fig. $26 \mathrm{ak}$ ) erinnern, wirklich gesehen zu haben glaube.

Was nun das Vorkommen solcher Kerne bei anderen Stemma's anlangt, so habe ich bisher allerdings nirgends mehr dergleichen $\mathrm{Ge}^{-}$ bilde mit der erforderlichen Sicherheit wahrgenommen, bedenkt man aber 1) dass ich deren Verbreitung bisher nicht, was entschieden nöthig ist, zum Gegenstand eines besondern Studiums machte, 2) dass die übrigen von mir untersuchten Stemma's, zumal die der einheimischen Myriopoden, relativ sehr klein sind, 
so ist es, wie wir schon oben hetonten, gewiss vi el wahrscheinlicher, dass diese Kernesich der Beobachtung entzogen haben, als dass sie wirklich fehlen ${ }^{1}$ ). Dass sich aber die Retinaschläuche des Tracheaten-Stemma's in der That nicbt in das Grenacher'sche Schema bannen lassen, zeigt auf das Eclatanteste der Umstand, dass ausser dem basalen Kern der Ganglienzelle, der von uns allgemein nachgewiesen ist, und ausser dem apikalen Nucleus, von dem ich auf Grund des Obigen annehmen muss, dass er gleichfalls eine weitere Verbreitung hat, auch noch und zwar zunächst bei Scolopendra (Fig. 18) ein dritter Kern zur Beobachtung gelangte.

Hier sahen wir nämlich oberhalb der basalen Anschwellung und ungefähr in der Mitte jedes Retinaschlauches einen deutlichen etwas länglichen Fleck (m k), von doppelter Kontur umgrenzt und im Innern, offenbar durch das ziegelrothe Augenpigment, roth gefärbt. Anfangs, obwohl aus später zu erörternden Gründen vom Vorkommen eines solchen Mittelkernes durchaus nicht uberrascht, dachten wir, dass hier vielleicht Schnitte durch quer oder auch etwas schief getroffene Retinalschläuche vorlägen; die ganze Lagerungsweise aber, ferner die Beschaffenheit sowie die intensive Pigmentirung und der Umstand, dass diese Gebilde beträchtlich schmäler $(0,005)$ als die Schläuche selbst (0,006-7) sind, lässt wohl kaum eine ungezwungenere Deutung als die zu, dass man es hier wirklich mit besonderen Inhaltskörpern zu thun hat, die ihrer Lage nach den von uns inzwischen nachgewiesenen Kernen der mediären Schichte im Alciopiden- und Eunice-Auge entsprechen widrden.

Andeutungen solcher Kerne fanden wir auch einmal an einem Zupfpräparat vom Scorpio-Seitenauge (Fig. $5 \mathrm{mk}$ ), sowie an den vorderen und hinteren Mittelaugen von Epeira (Fig. 25 u. Fig. $26 \mathrm{mk}$ ) und zwar im Anfang der Entfärbung durch Oxalsäure, doch bleibt hier z. Th. die Möglichkeit einer Verwechslung mit den Kernen der Ganglienzellen nicht ausgeschlossen ${ }^{2}$ ).

1) Unzweifelhafte präbacilläre Kerne (Fig. 29 ak) neben grossen basalen Nucleis (Fig. 27 und $28 \mathrm{gz}$ ) habe ich neuerlichst an frisch untersuchten Augen von Tegenaria domestica beobachtet. (Vgl. die Figuren-Erklärung.)

2) Neuestens gelang es uns an den in Fig. $26 \mathrm{mit} \mathrm{mk}$ ? bezeichneten Gebilden mit Alaun-Carmin eine sehr distincte Färbung zu erzielen. 
Hier muss ich noch speciell in Bezug auf das hintere Mittel(Scheitel-) Auge von Epeira einer anderen höchst auffallenden Differenzirung gedenken. An einem medianen, d. i. der Körperlängsachse parallelen Schnitte erscheint die Retina dieses Stemma's in der That in der von Grenacher (Fig. $18 \mathrm{~B}$ ) angegebenen Art, wobei gegenüber dem Vorderauge besonders der Umstand bemerkenswerth ist, dass anscheinend sämmtliche Opticusfasern ohne die geringste basale Anschwellung in die stäbchentragenden Endschläuche (vergl. auch unsere Figur 26 rechts) übergehen und damit also gegenüber dem gewöhnlichen Verhalten eine nicht $\mathrm{zu}$ verkennende Abweichung begriinden. Schneidet man aber das erwähnte Hinterauge transversal und zugleich etwas schief von hinten und oben nach vorne und unten durch, so zeigt sich (Fig. 26) folgender Sachverhalt. Circa zwei Drittel der ganzen Retina, und zwar der gegen die Körper-Mittelaxe gerichtete Theil, zeigen die vorerwähnte Pallisadenstructur (st). Der iibrige Abschnitt aber, also der im äusseren Winkel gegen das seitliche Integument ( $\mathrm{hp}$ ') gelegene, bietet eine völlig andere Beschaffenheit. Auf den ersten Blick erscheint das Ganze als eine Anhäufung (0,01 mm) dicker Ganglienzellen (gz) mit sehr deutlichen $(0,004-5$ grossen) Kernen, die jenen des Vorderauges (Fig. $25 \mathrm{gz}$ ) sozusagen identisch sind. Zunächst könnte man nun glauben, dass diese Gebilde zu den rechts gelegenen Stäbchen-Schläuchen gehören, dass aber hier das Gánglienzellenlager ausnahmsweise ganz seitwärts zusammengedrängt sei.

Diese Erklärung widerspricht aber einmal dem leicht zu konstatirenden Verhalten der Bacillärschläuche, welche nach hinten unmittelbar in die Opticusfasern tibergehen, und dann der Beschaffenheit gedachter Zellen selbst. Man sieht nämlich, dass sie sich gleich jenen des Vorderauges nach oben zu schlauchartig verlängern, wobei aber diese peripherischen Fortsätze, so viel wir bisher erkannt haben, gegen einen mittleren in der Höhe der Stäbchen gelegenen hellen und anscheinend sphärisch begrenzten grossen Körper ron 0,026 mm Durchmesser zusammenneigen. Da letzterer $(x)$ in mancher Hinsicht an die subcuticulare und von einer schmalen Epithelschicht (Matrix?) umgebene Linse des Raupenanges erinnert, und auch einzelne kleine Kerne sowie ein stellenweise stärker pigmentirter Hof um diesen Körper sicht- 
bar sind, so frägt man sich unwillktirlich, ob denn hier inner halb des allgemeinen Augen rahmens und unterhalb des gemeinsa men Glaskörpers eine Art subordinirtes Binnenange eingeschaltet sein soll.

Dass es mit dieser höchst eigenthlimlichen Differenzirung eines Abschnittes der Gesammt-Retina wirklich seine Richtigkeit habe, beweist auch der Umstand, dass man am andern Auge des betreffenden (Hinter-) Paares und zwar an der korrespondirenden Stelle, d. i. wieder an der äussern Seite, genau dieselbe Bildung antrifft ${ }^{1}$ ).

Zuletzt noch eine Bemerkung beztiglich der Endausbreitung der Opticusfasern.

Während sich nach Grenacher die Retinaschläuche in der Regel als ziemlich gerade Fortsetzungen der Fasern des Opticus darstellen und die retinalen Abschnitte der letzteren gewissermassen nur durch die Anschwellung ihrer Enden etwas auseiandergebogen werden, findet sich speciell am Seitenauge von Scorpio entschieden eine besondere retinale Faserlage ausgebildet. Wie Fig. 4 lehrt, breitet sich der Sehnerv am Grunde der Retina zu einem ziemlich dicken, der Sclera-Matrix anliegenden, schalenförmigen Stratum aus, von dem dann unter z. Th. sehr beträchtlichen Winkeln die einzelnen zu den Ganglienzellen hintretenden Fasern abzweigen. Es zeigt sich hier demnach ein ähnliches Verhalten wie etwa am Retinabecher der Wirbelthiere oder auch an den Otocysten, wo aber die Faserschichte des Acusticus eine complete Kugelschale bildet.

1) $\mathrm{Ob}$ in dieser seitlichen Zweitheilung der Retina bez. auch des zugehörigen Opticusfaserstranges vielleicht irgend eine Analogie mit gewissen von Grenacher erwähnten Befunden an den angeblich stäbchenlosen VorderSchläuchen im Vorderauge von Lycosa (p. 49, Fig. 22 A) und den gleichfalls abacillären Randstrahlen von Salticus (Fig. 28) vorliegt, muss vorläufig dahingestellt bleiben; genug, dass wir sehen, dass sich das Princip der Arbeitstheilung nicht bloss an die einzelnen Augen eines und desselben Thieres, sondern auch auf die einzelnen von vornherein doch wahrscheinlich homologen bez. homonomen Strahlstücke einer und derselben Retina erstreckt. 


\section{Axialgebilde der Retinaschläuche.}

Nach Grenacher sind die eigentlichen Axenstäbchen solide und zwar im Allgemeinen theils cylindrische, theils prismatische oder auch plattenförmige Einlagerungen vorwiegend im vordersten $A b$ schnitt der Endschläuche, die nach hinten derart scharf abgeschnitten sind, dass (p. 158) „für einen directen $Z$ u sa mmenhang zwischen dem Stäbchen und der Nervenfaser (d. h. mit Ausschliessung der Vermittlung durch die Zelle), b is her noch alle Indicien fehlen", womit offenbar, wie der Context zeigt, vornehmlich die Abwesenheit eines u. A. auch von $R$. Greeff am Alciopidenauge angegebenen Axenfadens betont werden soll.

Eine solche scharfe hintere Abgrenzung hat ibrigens Gre$\mathrm{n}$ acher im Ganzen nur in relativ wenigen Fällen constatirt, nämlich bei Phalangium (Fig. 16, 17), dann am Vorder- sowie am Hinterauge von Epeira (Fig. 19), ferner bei Lycosa (Hinterange) und endlich bei Musca vomitoria, deren Mittel- d. i. in die Augenaxe fallenden Stäbchen länger als die Randelemente wären.

Bei den übrigen Stemma's dagegen blieb das Stäbchenhinterende ziemlich unbestimmt. Abgesehen davon, dass, wie bei der Ungunst der Objecte leicht erklärlich, bei Pulex canis (Fig. 29) nur ein ,gezähnelter Rand" angegeben wird, und dass am Vorderauge von Lycosa (p. 49 Fig. 22 A) nur an der Hinterpartie nach vorne an Grösse abnehmende, hinten spitz zapfenartige kurze Stäbchen bemerkt wurden, gilt dies besonders von den Salticusund den Wespen-Augen.

An den ersteren (äusseres Vorderrandauge und hinterstes) sieht man auf den betreffenden Grenacher'schen Figuren (25 u. 27) auch nicht die Spur einer hinteren Grenze und bemerkt auch Grenacher (p. 51): „Nach hinten hin wird es schwieriger zu sagen, wie weit sie reichen, weil sie allmälig an Lichtbrechung abnehmen und dafür die den Retinazellen angehörige Granulirung ebenso allmälig einsetzt."

Höchst interessant wäre nach ihm auch bezüglich der Stäbchen-Entfaltung das innere Vorderrandauge (Fig. 28). Die Schläuche der Mantelschichte wären, gleich jenen von Phryganea grandis überhaupt, stäbchenlos. Die nächst folgenden innern Schläuche hätten dann vorne kurze scharf umgrenzte Zapfen, während die Stäbchen 
der innersten axialen Schläuche („Mittelzellen“) sehr dïnn und zart erschienen und sich sehr stark gegen den Opticus (nach der Zeichnung gegen die ganglienzellenartige Basalanschwellung) hin verlängern (p. 54). Nach dieser letzteren Darstellung wird Grenacher selbst zugeben, dass für einen ev. engeren Zusammenhang der Stäbchen mit den specifischen Nervenelementen doch nicht "alle Indicien fehlen".

Uebergehend auf die eigenen Untersuchungen so beziehen sich dieselben vornehmlich nur auf die Stäbchen im Scorpionidenauge, beztiglich welcher wir dem Leser mehrere Ansichten vorführen.

Fig. 12 zeigt zunächst ein Zupfpräparat aus einer frischen Retina und zwar vom Mittelange des Scorpio aus. Man sieht hier ein Bündel von Retinalschläuchen, aus deren abgerissenen Vorderenden die Stäbchen als griffelartige und an der Spitze etwas hackig umgebogene Gebilde herausragen.

Ein ähnliches Bild zeigen uns auch die betreffenden Elemente an Schnitten durch die Seitenaugen des gleichen Scorpions (Fig. 4 und 5), nur dass man hier, falls das Präparat behatsam mit Salpetersäure entfärbt wird, die hinteren Enden der Stäbchen weiter zurück verfolgen kann, wobei sie aber selbst an fein zertheilten Zupfpräparaten schon beträchtlich vor der Ganglienzelle dem Auge entschwinden.

Einige bemerkenswerthe Aufschlüsse gibt betreffs desselben Auges noch der ziemlich oberflächliche Retinaquerschnitt in Fig. 8, der die Endschläuche selbstverständlich in sehr verschiedener Höhe und Richtung trifft. Man sieht hier (z.B. ez, eza), 1. dass die völlig soliden Stäbchen nicht der Länge nach zusammengesetzt, sondern einfach sind, und 2. dass sie wenigstens mit ihren vorderen Abschnitten den deutlich doppelkonturigen Schlauch bis auf einen geringen eine pigmentirte körnige Substanz enthaltenden Zwischenraum fast vollständig ausfüllen, während sie nach hinten sich etwas zu verschmälern scheinen.

Den besten Einblick gibt nun aber der mehr erwähnte halb zerzupfte Retinalschnitt von Buthus in Fig. 14. Hier erscheinen die „Stäbchen" als dicke homogene Axenstränge, die ca. nur ein Drittel der Schlauchbreite messen, und sich bis hart an die Ganglienzelle zurück verfolgen lassen. Meist zeigt dieser Strang einen mehr oder weniger welligen Verlauf und erinnert so an die Bilder, welche Leydig, auf Taf. IX Fig. $3 \mathrm{c}$ 
vom Stemma der Honigbiene zeichnet. Dass aber dieser Mittelstrang hier nicht etwa (wie Leydig annimmt) auf die verdickten Kanten der Schläuche bezogen werden kann, zeigt einmal der runde Querschnitt der letzteren und dann der Umstand, dass ich denselben an abgerissenen Schläuchen mit aller Sicherheit als ein selbständiges inneres Gebilde erkannte. Da namentlich der hintere Abschnitt dieser Stränge bisweilen ein feinstreifiges Aussehen zeigt und an einzelnen Schläuchen wenigstens aus einem besonderen zipfelartigen Fortsatz der Ganglienzellensubstanz zu entspringen scheint, so dürte die Anschaung, dass man es hier mit dem eigentlichen peripherischen Nervenende zu thun hat, doch wohl eine nenerliche Prüfung verdienen.

Vergleichung der stemmalen Retinastrablen mit den Endschläuchen der tympanalen Sinnesapparate der Orthopteren.

Schon in meiner Monographie der letztgenannten Organe habe ich wiederholt auf die Aehnlichkeit zwischen denselben und den stemmalen Sehwerkzeugen sowohl hinsichtlich des gesammten Bauplanes als mit Rücksicht auf die morphologische und histologische Beziehung ihrer Endgebilde hingewiesen und nunmehr, da ich auch das andere Vergleichungsobject aus eigener Anschauung näher kenne, bin ich in der Lage diese Parallele noch weiter auszuführen.

Die Confrontirung des Müller'schen Tympanalorganes der Acridier (etwa nach Fig. 134 des gedachten Werkes) mit einem Stemma z. B. dem von Scolopendra in Fig. 17 a ergibt zunächst die allgemeine Uebereinstimmung, indem speciell die Anordnung der basalen Ganglienzellen und die der beidemale zu einem Becher vereinigten stäbchentragenden Endschläuche bei beiden Organen so zu sagen die gleiche ist.

Zur Vergleichung im Detail diene dann der beistehende Holzschnitt. 


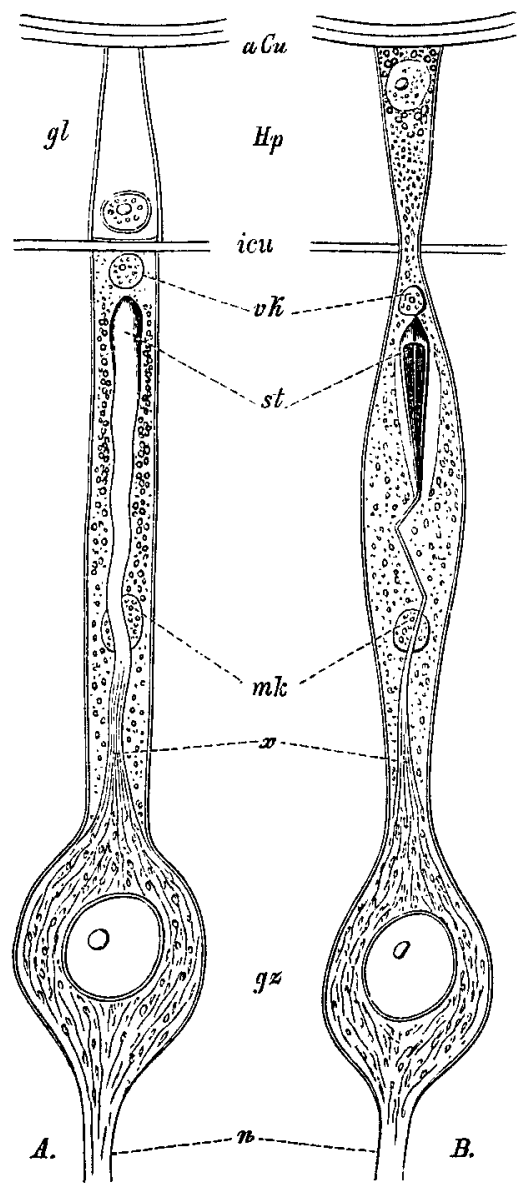

A. Schema eines retinalen Endschlauches von Buthus. B. Schema eines tympanalen Endschlauches von Acridium. aCu äussere Cuticula, Hp Hypodermis, gl Glaskörper, icu innere Cuticula, $v \mathbf{k}$ Vorderkern des Endschlauches, st Stäbchen, mk Mittelkern, x Axenstrang (Chorda), gz Ganglienzelle mit Kern. n Opticus- resp. "Austicus"-Faser.

A ist eine nur wenig vereinfachte Copie eines Retinalstrahles von Buthu s in Fig. 14; B desgl. von einem „Hörschlauche", nach den Figuren 132, 84, 90 und 92 in genannter Abhandlung. An letzterem findet man nach wiederholten und genauesten Untersuchungen: $\mathrm{Zu}$ änsserst die Cuticula $(\mathrm{aCu})$, darunter eine deutliche Hypodermzelle $(\mathrm{Hp})$ mit einem besonderen Kern, in der Regel, wie an den Archiv f. mikrosk. Anatomie Bd. 17. 
meisten Matrixzellen, von Pigment umgeben. Diese zieht sich (vgl. Figur 86 und 132) in einen mehr weniger langen Fortsatz aus, der scheinbar oft ohne Grenze (icu) in den eigentlichen spindelförmig aufgetriebenen Endschlauch iubergeht. Nach Innen verengert sich dieser Schlauch und erweitert sich dann bald früher bald (Locustiden Fig. $74 \mathrm{vN}, \mathrm{gz}$ ) erst in sehr beträchtlicher Entfernung wieder zur grossen kugelartigen Ganglienzelle, die einen umfangreichen doppelteontourigen Kern mit 1-3 deutlichen Kernkörperchen einschliesst.

Im Wesentlichen dieselbe Gliederung zeigt nun auch nach dem Obigen der Retinastrahl des Stemma's, nur dass hier die (axiale) Integumentzelle nicht pigmentirt, sondern vollkommen durchsichtig erscheint, und ferner vom Endschlauch durch die innere integumentale Grenzhaut scharf abgegrenzt ist. Dass aber auch hier der sub-integumentale Strahl-Abschnitt in zwei scharf gesonderte Strecken, nämlich in die Ganglienzelle und in den eigentlichen Endschlauch gegliedert ist, dürfte nach dem früheren kaum zu bezweifeln sein.

Betreffs des wichtigsten Bestandtheiles, nämlich des stäbchenartigen Axengebildes ist dann dies zu beachten. Die in Fig. 84 und 90 des genannten. Werkes abgebildeten mit grösster Sorgfalt im frischen Zustand präparirten Hörschläuche (resp. Stifte) beweisen, dass hier der dünne chordaartige Axenstrang entschieden mit mehreren nach aussen convergirenden Fasern $(w)$ in der Ganglienzelle selbst wurzelt und demnach als directe Fortsetzung eines wahren Nervenelementes aufzufassen ist.

Wenn nun auch a priori die Sicherheit der betreffenden Beobachtungen nicht den weniger exacten Befund hinsichtlich des Axenstrangs der Buthus-Retina zu ergänzen vermag, so zeigt sie doch, dass es factisch so sein kann, während für di e Annabme, dass der Axenstrang hier nicht aus der Ganglienzelle entspringt, sondern als Erzeugniss oder Abscheidungsproduct des Endschlauches selbst frei in demselben schwebe, kein sicherer Anhaltspunct zu finden ist. Da es übrigens bezïglich der stiftartigen Endanschwellung an der Axenfaser des Hörschlauches zweifelhaft bleibt, ob dieser Kolben in seiner Totalität als Endtheil der Axenfaser aufzufassen ist, oder ob bloss sein Mittelfaden als solcher zn gelten hat, während der tibrige oder Manteltheil vielleicht eine cuticulare Abscheidung des 
Endschlauches vorstellt, so ist die Möglichkeit, dass auch der Sehstab, wie ihn Grenacher bei manchen Spinnen darstellt, von dem, was wir bei Buthus als Axenstrang bezeichnen, verschieden sei, immerhin in Erwägung zu ziehen.

Einen besonderen Nachdruck lege ich aber speciell auf die Uebereinstimmung in den Kerngebilden der zwei Arten von Endschläuchen.

An sämmtlichen Hörschläuchen findet man mit $\nabla$ ollster Sich e rhe it (ausser dem Ganglienzellen-Nucleus) wenigstens noch einen theils mehr basal, theils median gelegenen Kern (Fig. 85, 84 etc. wk), der der Lage nach mit dem Mittelkerne (mk) von Scolopendra) ubereinstimmt; und die Frage, ob der Gesammtendschlauch (incl. Ganglienkugel) als einzelliges Gebilde betrachtet werden kann, muss sonach hier mit aller Entschiedenheit verneint werden.

An den schlanken Hörschläuchen mancher Orthopteren und ganz allgemein an den Endblasen des Siebold'schen Tibialorganes der Locustiden findet man aber ausser dem „Wurzelkern" (wk) auch noch einen "Gipfelkern" (gk), der offenbar mit dem präbacillären Nucleus der Retinaschläuche zu vergleichen wäre.

Während nun, wie wir schon gehört, Grenacher den gesammten Retinastrahl als eine einzige einkernige Zelle auffasst, ferner die höchst auffallende ungleiche Lage des Kernes (p. 55) durch eine Verschiebung in Folge der Stäbchenausscheidung erklärt und (p. 49) noch ausdrücklich hervorhebt, dass zwischen der Opticusfaser und der „Retinazelle" keine Einschiebung eines neuen histologischen Elementes (Ganglienzelle) stattfindet, erweist sich uns nach dem vorstehenden das stemmale so gut wie das tympanale Endgebilde als ein in zwei Abschnitte, Ganglienzelle und Endschlauch differencirtes Organ, und da, z. Th. wenigstens, der Endschlauch selbst wieder zwei Kerne besitzt, muss man wohl Anstand nehmen, selbst diesen Abschnitt allein als ein einfaches Zell-Individum zu betrachten.

\section{Parietale Pigment- und Matrixzoneder Retina.}

Behandelt man die Retina von Scolopendra mit Kalilauge, so sieht man, dass dieselbe zweierlei auch der Farbe nach ver- 
schiedene Pigmentschichten enthält, nämlich (Fig. 18) eine allgemeine parietale Zone von (nach Kalizusatz) blauvioletter Farbe, die sich auch auf den Opticus fortsetzt und iberhaupt an die $\mathrm{Ma}$ trix der Sclera bez. der cuticularen Nervenscheide gebunden ist, und dann ein im frischen Zustand mehr kirschrothes später ins Ziegelrothe übergehendes Pigment, das die einzelnen Sehstäbe einhüllt.

Eino eigenthümliches und nicht ganz leicht zu erklärendes Verhalten bietet nun besonders die parietale Pigmentzone der $\mathrm{Bu}$ thus-Retina.

An einem unentfärbten Axenschnitt (Fig. 13 links) bemerkt man zunächst, dass das dunkelschwarze Pigment derart vertheilt ist, dass es eine zusammenhängende auch die Vorder-Wand der Retina in sich begreifende Parietalzone bildet, während es in der mittleren von den radiären Schläuchen eingenommenen Sehichte eine streifenweise Anordnung zeigt.

Nach längerer aber schonender Einwirkung von Kalilauge verblasst dann die Mittelzone (Fig. 13 rechts und Fig. 14) vollständig, während ringsum, auch vorne, also an der Linsenseite, ein breiter rosagefürbter Sanm zuruckbleibt. Mustert man nun diesen zunächst an der Hinterseite bei starker Vergrösserung, so erweist er sich als eine feinkörnige Lage mit einzelnen meist nicht sehr deutlichen Kernen (Fig. $14 \mathbf{k}^{\prime \prime \prime}$ ). Da man nun ausserdem in dieser granulären Schichte quere und auf der Sclera senkrecht stehende Züge resp. Scheidewände bemerkt, so ist die Annahme, dass man es hier mit einer hohen cylinderepithelartigen Sclera-Matrix zu thun habe, von vorne herein gewiss sehr naheliegend. Auffallend ist aber, dass der Vordersaum der Retina, unter dem Septum, die, trotz der an dieser Stelle liegenden Kerne doch kaum als ein selbständiges Matrix-Epithel angesprochen werden kann, wenigstens hinsichtlich der Färbung doch anscheinend kontinuirlich in die Hinterschichte übergeht.

Untersucht man nun vorerst die Seitentheile ( $\left.\mathrm{k}^{\prime \prime}\right)$ dieser pigmentirten Mantelschicht und zwar zunächst weiter oben vom Kelchrand $\left(k^{\prime}\right)$ aus, so scheint ein solcher Uebergang factisch auch in anderer Beziehung stattzufinden. Hier sieht man nämlich deutliche mit einem sehr distincten Nucleus versehene Zellen, resp. Endtheile von solchen, die aber schon insoferne kaum als einfache Wandepithelelemente gelten können, als sie auf der zugehörigen 
Cuticula (Sclera) nicht senkrecht, sondern sehr schief stehen, so zwar, dass siegegenden Kelchrand zu sichallmälig in gleiche Linie mit den stäbchenführenden Retinalschläuchen stellen.

Beachtet man nun ferner: 1. dass diese Seitenwandelemente nach oben stufenweise länger zu werden scheinen, 2. dass man an ihrer Basis in der Regel stark lichtbrechende auf Stäbchen zu beziehende Einschlüsse findet und 3. endlich den Uebergang in die gerade nach vorne verlaufenden Retinalschläuche, so kommt man zur Ueberzeugung, dass sie selbst nichts anderes als die durch den Schnitt abgetrennten Endstücke von solchen sein hönnen, die aber, da andere subcuticulare Gebilde völlig zu fehlen scheinen, jedenfalls, wenigstens auf dieser Strecke, unbeschadet ibrer allfälligen anderen Functionen die Rolle von Matrixzellen übernommen haben.

Im Gegensatz zu Grenach er, der einerseits sämmtliche Retinalschläuche gegen die Linse streben lässt, während er andererseits den schon oben berihrten und gleichfalls nach innen convergirenden „Randzellen" (z. B. seine Fig. 28 Rt') die Stäbchen z. Th. abspricht, sehen wir also bei Buthus und - wenn auch weniger klar - bei Epeira (Fig. 25 ak), dass die äussersten Retinaschläuche schon weit unten an der Seitenwand der Augenkapsel endigen, während die übrigen, in stufenweiser Anordnung, ihr Ende nur successive nach oben, bez. nach innen gegen den Boden des Retinalkelches verlegen. $\mathrm{Da}$ es von vorne herein zum mindesten etwas ungewöhnlich erscheint, dass die Enden der Retinaschläuche im Dienste der Cuticular-Bildung stehen sollten, möchten wir noch Folgendes hervorheben. Aus Fig. 13 (links) ergibt sich, dass auch die zur Linse hinstrebenden Randschläuche kaum eine perceptive Bedeutung haben können, da durch das weit darïber hinaus, d. i. nach innen sich verbreitende Hypodermis- oder Irispigment (Ir) das Licht vom Retinarande abgeblendet wird, und konnten also die betreffenden stets im dunkeln stehenden Elemente z. Th. vielleicht unter totaler oder doch partieller Rückbildung der Stäbchen einer anderen Function ganz wohl angepasst werden.

Retinula-artige Gruppirung der Netzhautstrahlen im Scorpionstemma.

Einen Hauptunterschied zwischen dem unicornealen Stemma 
und dem multicornealen oder Facettauge findet Grenacher besonders in der Lagerungsweise ibrer Retinaelemente, nämlich darin, dass diese beim ersteren als morpho- und physiologisch vollständig selbständige, pallisadenartig nebeneinander stehende letzte Einheiten der Retina erscheinen, während sich die correspondirenden und von Grenacher als einfache Zellen betrachteten Elemente im Netzauge zu Einheiten höherer Ordnung d. i. zu den sog. Retinulae vereinigen oder gruppiren, indem letztere nach den grundlegenden und ansgedehnten Nachweisen Grenacher's, thatsächlich immer aus einer mehr oder weniger innigen "Coalescenz einer bestimmten Anzahl (meist 7, dann 5 und 4, selten 8) von Einzelelementen" (p.137) hervorgehen, wobei bekanntlich in der Regel die gegen die Axe der ganzen Zellgruppe geriuckten Sehstäbe zu einem einzigen mehrtheiligen oder gemeinschaftlichen Axenstab, dem „Rhabdom" verschmelzen.

Dass aber Grenacher die völlige Is olirtheit der einzelnen Retinaenden im typischen Stemma auch als eine ganz allgemeine Eigenschaft dieser Augen auffasst, geht dann aus einer Stelle (p. 138) hervor, wo er gegen die von Leydig betonte Vergleichbarkeit des Stemma's mit dem Facettange (in toto) auf die dem letztern so "eigenthümliche" dem Stemma aber "fehlende" „Gruppenbildung" hinweist, und dann weiter meint, dass demnach auch ohne Dazwischentreten eines .,Deus ex machina" an eine Umwandlung des einen Auges in das andere im Sinne der Descendenztheorie nicht gedacht werden könne.

Um so interessanter dürfte dem gegenüber der folgende Nachweis sein, dass in der That auch ein typisches Stemma, und zwar das des Scorpions, eine parcellirte oder, wenn wir so sagen dïrfen, eine facettirte Netzhaut besitzt. Bereits im Herbst 1875 erkannte ich auf der napfartig ausgehöhlten Vorderfläche einer frischen Retina eines Mittelauges von Scorpio europ. unterhalb des Glaskörper-Epithels (gl) die auf der aus jener Zeit stammenden Figur 6 dargestellte höchst auffallende Mosaik aus konstant fü nfstrahligen Rosetten (r, ro und Fig. 10, 11) und tiberzeugte mich an reichem frischen Material, das mir der bekannte Arachnologe A. A usserer verschaffte, dass die Strahlen dieser Rosetten nichts Anderes sind als die an ihrer Spitze hakenartig nmgebogenen Stäbchengebilde (Fig. 9, 12).

Diese wichtige Thatsache trug ich dann zuerst in der Januar- 
sitzung der zool.-bot. Ges. in Wien (1876) vor, wobei ich die Rosetten mit den mehrtheiligen Endkolben (des Sehstabes) von Herbstia (Leydig, Histologie p. 251 Fig. $133 \mathrm{~A}$ ) verglich.

Ausserdem erwähnte ich noch dieses Factum in meinem Buch „Organismus der Insecten" (München 1877), wo es p. 286 heisst: „Eine merkwürdige Erscheinung haben wir schon vor längerer Zeit an den Scorpionaugen entdeckt. Hier sondern sich die aus mehreren Körner- und Faserlagen (Ganglienzellen- und Opticusfaserschichte!) sich erhebenden Sehstäbe in Gruppen von je fünf Individuen."

Neuestens habe ich mich nun, unter gleichzeitiger Revidirung der älteren Präparate ïberzeugt, dass diese Gruppirung keineswegs bloss auf die Mittelaugen beschränkt, sondern in ganz gleicher Weise auch den Seitenaugen eigen ist, worüber man den Flächenschnitt Fig. 8 einsehe. Auch hier findet man stets nur pentamere Rosetten und bemerkt zugleich, dass die bei der höchsten Einstellung ganz kurzen, beim Senken des Tubus aber sich verlängernden d. i. auf grössere Tiefen hinab sichtbar werdenden Stäbe bis zur Spitze hin von einem besonderen Futteral, d. i. eben vom Retinalschlanch umbïllt sind.

Dass nun aber diese (bei der tiefsten Einstellung ca. 0,03 mm breiten) Gruppen wirklich den Retinulae des Facettauges entsprechen, zeigt am Besten die Vergleichung z. B. mit der TipulaRetina (Grenacher's Fig. 44, 45), wo man in der That ganz ähnliche, aber bekanntlich siebenstrahlige Stäbchenrosetten wahrnimmt.

Ungewiss bleibt mir aber, ob diese Endzellen- resp. Stäbchengruppen durch besondere Pigmentzonen von einander isolirt sind. Auf dem Präparat Fig. 6 und 8 finden sich Andeutungen davon und auch der Tiefenschnitt Fig. 13 (links) würde dem nicht widersprechen; es fehlt mir aber gegenwärtig das Material, um diese wichtige Frage endguiltig zu lösen.

Da die stemmalen Stäbchen in der Regel mehrtheilig, die von Scorpio aber entschieden einfach sind, so ergibt sich von selbst die Frage, ob zwischen dem polymeren Stäbchen e in er Endzelle und dem rhabdom-artigen Stäbchencomplex einer ganzen Zellgruppe eine gewisse functionelle Uebereinstimmung besteht.

Auf alle Fälle hat man es hier mit einem hochinteressanten Fall von Convergenzbildung zu thun; da ron einen unmit- 
telbaren Uebergang eines so hoch differencirten Stemma's in ein Facettange in der That nicht wohl die Rede sein kann.

\section{Hanptergebnisse.}

1. Die Cornea-Linse des Stemma's zeigt nicht bloss die lamellare Structur, sondern auch die feinen Porenkanäle der integumentalen Chitin-Cuticula.

2. Die Retina des Stemma ist in ihrer ganzen Ausdehnung durch eine besondere cuticulare mit der Sclera zusammenhängende Zwischenlamelle (präretinales Septum) vom integumentalen Epithel (Hypodermis, „Pigment-" und „Glaskörper"zellen) abgesondert. - Dies spricht (vom rein topographischen Standpunct aus) für die Ausschliessung derselben von der Hypodermis.

3. Der einzelne Retinastrahl des Stemma ist im Allgemeinen keine einfache Zelle (Grenacher), wie jener des Facettauges, sondern gliedert sich in zwei Abschnitte, in eine basale Ganglienzelle und in einen ein- (vielleicht $z$. Th. auch zwei-) kernigen Endschlauch. Dies spricht gegen die unmittelbare Vergleichbarkeit des ge sam m te $n$ stemmalen Retinastrahles mit den "Retinazellen" des Facettauges.

4. Der Axenstab der Retinaschläuche von Buthus scheint eine directe mediane Fortsetzung der Ganglienzelle, resp. der Opticusfaser zu sein.

5. Die Retinaschläuche von Buthus convergiren keineswegs alle gegen die Linse $\mathrm{zu}$, sondern die äussersten endigen schon tief unten an der Wand der Augenkapsel und rïcken stufenweise gegen den Kelchrand resp. gegen die obere Fläche des Retinapolsters empor.

6. Die Enden der Retinaschläuche scheinen, z. Th. wenigstens, die Matrix der Sclera zu bilden.

7) Die Retinalschläuche des Buthns-Stemma sind nicht isolirte Elemente, sondern pruppiren sich, wie im Facettauge, zn pentameren (retinula-artigen) Perceptionsorganen höherer Ordnung. 
Grössen-Angaben in Millim.

\begin{tabular}{|c|c|c|c|c|c|c|c|c|c|}
\hline & \multicolumn{2}{|c|}{ Linse } & \multirow[b]{2}{*}{$\frac{b}{d}$} & \multicolumn{2}{|c|}{ Glaskörper } & \multicolumn{2}{|c|}{ Ganglienzelle } & \multicolumn{2}{|c|}{ Endschlauch } \\
\hline & 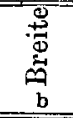 & d: & & 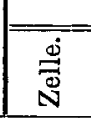 & $\underset{d}{\Delta}$ & $\frac{\dot{g}}{\stackrel{9}{9}}$ & 过 & 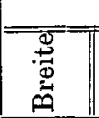 & 这 \\
\hline $\begin{array}{l}\text { Scorpio eur. } \\
\text { Seitenauge }\end{array}$ & 0.22 & 0.28 & 0.7 & 0.006 & & 0.015 & 0.01 & & \\
\hline $\begin{array}{c}\text { Buthus afer } \\
\text { Mittelauge }\end{array}$ & 0.41 & 0.30 & 1.4 & 0.008 & 0.006 & 0.017 & 0.01 & 0.01 & 0.005 \\
\hline $\begin{array}{c}\text { Epeira Schreib. } \\
\text { Vorderauge } \\
\text { Hinterauge }\end{array}$ & 0.20 & 0.15 & 1.3 & & 0.006 & $\begin{array}{l}0.01 \\
0.01\end{array}$ & $\begin{array}{l}0.006 \\
0.006\end{array}$ & $\begin{array}{l}0.006 \\
0.006\end{array} \mid$ & 0.004 \\
\hline Julus sabul. & 0.05 & 0.08 & 0.6 & $\begin{array}{c}0.008 \\
\text { Dicke. }\end{array}$ & & 0.005 & & & \\
\hline Scolopend. cing. & 0.23 & 0.22 & 1.0 & 0.011 & & & $0.008 ?$ & 0.007 & \\
\hline Lithobius forf. & 0.07 & 0.07 & 1.0 & 0.008 & & 0.008 & & & \\
\hline
\end{tabular}

Erklärung der Abbildungen anf Tafel V bis VII.

Durchgehende Bezeichnungen.

$\mathrm{aCu}$ äussere Cuticula.

c-l Cornea-Linse.

$\left.\begin{array}{l}P \text { weite } \\ p \text { feine }\end{array}\right\}$ Cuticula-Poren.

hp Integumental-Epithel, (Hypodermis).

pz Linsen-Rand- oder Pigmentzellen.

gl Linsenepithel- oder Glaskörperzellen.

gr innere cuticulare Grenzhaut der Hypodermis.

sc Fortsetzung der Grenzhaut auf die Retina (Sclera).

la Fortsetzung der Grenzhaut auf die Vorderfläche der Retina (präretinale Zwischenlamelle oder sog. Hyaloidea). 
$\checkmark$ Spaltungs- resp. Verbindungsstelle der genannten drei Grenzhautstrecken.

fa Opticusfasern.

gz Ganglienzellen der Retina.

gk Kern derselben.

ez Endschläuche (der Retina).

ak apicaler oder Vorderkern.

mk Mittelkern derselben.

st Stäbchen.

ax Axenstrang bez. Fortsetzung der Stäbchen nach innen gegen die Ganglienzelle.

no nervus opticus.

Tafel $\nabla$.

Fig. 1. Scorpio europaeus Schr. Mittelaugen isolirt. r Retina. no nerv. opticus. Schwach vergrössert.

Fig. 2. Scorpio europaeus Schr. Seitenaugen von innen. Schwach vergr.

Fig. 3. $n n$ Partie eines transversalen Tiefenschnittes durch die Mittelaugen. $\mathbf{r}$ Retina in toto. $\mathbf{r}^{\prime}$ im Durchschnitt. b Blutgefäss in der integum. Bindegewebslage zwischen den Augen. $\mathrm{m}$ dorsoventrale Thoraxmuskeln. Alc. Kalilauge. Schwach vergrössert.

Fig. 4. Scorpio europaeus Schr. Seitenauge. Medianschnitt. Alcohol. Kalilauge. Camera lucida. Zeiss Immers. III. Die Retina zeigt deutlich drei Straten: Faser-, Ganglienzellen und Stäbchenzellenschicht. Vergr. $\frac{220}{1}$.

Fig. 5. Scorpio europaeus Schr. Retina-Zupfpräparat ebendaher. Man sieht deutlich den Vorderkern (ak) und Andeutungen eines Mittelkernes (mk). Zeiss Immers. III Vergr. $\frac{1000 .}{1}$.

Fig. 6. Scorpio europaeus Schr. Mittelauge. Frische mit Kalilauge behutsam entfärbte Retina von oben gesehen. Man erblickt die rosettenartig gruppirten Stäbchen r. ro. gl ein Stück hängengebliebenes Glaskörper-Epithel. Zeiss F.

Fig. 7. Scorpio europaeus Schr. Mittelauge. Stück eines Schnittes (f. $\left.3 r^{\prime}\right)$ nach starker auflösender Einwirkung von Kalilauge.

Fig. 8. Scorpio europaeus Schr. Seitenauge. Stück eines RetinaFlächenschnittes. Gegen die Mitte zu sieht man die zu fünfstrahligen Rosetten vereinigten Stäbchenenden, gegen den Rand quer- und schiefgetroffene Endschläuche, sowie zu äusserst auch Ganglienzellen gz. Alcohol. Kalilauge. Zeiss Immers. III. Vergr. $\frac{1000}{1}$. 
Fig. 9-12. Scorpio europaeus Schr. Mittelauge. Verschiedene Ansichten der Stäbchen-Gruppen von frischen Augen.

Fig. 9. Eine Gruppe halb von der Seite gesehen. Man sieht die hakenartige Umbiegung der Stäbchenspitze.

Fig. 10. Von oben bei hoher, Fig. 11 bei tiefer Einstellung.

Fig. 12. Frische isolirte Endschläuche mit den hakenartigen Stäbchenenden. Zeiss. Immers. II.

Fig. 13. Buthus a fer L. Mittelauge. Medianschnitt. Cam. lucida-Zeichnung links im natürlichen, rechts im entfärbten Zustand. Man beachte das hypodermale Randpigment (Iris Ir.), dann den dunkeln (rosafarbigen) Saum um die Retina (rechts), sowie die Kerne der Ganglienzellen (gz). h Spaitraum in der Cornealinse. Zeiss Imm. III. Vergr. $\frac{120}{1}$.

Fig. 14. Buthus afer L. Die äussere Partie (rechts) desselben Schnittes bei sehr starker Vergrösserung. $\frac{1000}{l}$.

\section{Tafe 1 VI.}

Fig. 15. Scolopendra cingulata L. Seitentheil des Kopfes mit den Augen.

Fig. 16. Scolopendra cingulata L. Transversalschnitt (in der Richtung $x \times$ Fig. 15) im unentfärbten Zustand. Man beachte den ziemlich dicken Glaskörper (gl) und das sich auf den Sehnerv (no) fortsetzende Netzhautpigment mit der relativ hellen Mittelzone (d). Vergr. $\frac{70}{1}$.

Fig. 17. S colopendra cingula ta L. Dasselbe bei stärkerer Vergrösserung. Zeiss Imm. L. cam. luc. nach Entfärbung mit Kalilauge. Retina des äusseren Auges (a) in toto mit durch den Linsenboden durchscheinendem Glaskörper; die des inneren (i) im Durchschnitt (Glaskörper zu schmal gezeichnet). Vergr. $\frac{160}{1}$.

Fig. 18. Scolopendra cingulata L. Randpartie desselben Retinaschnittes. k Kern der nach Kalilaugezusatz tiefblau sich färbenden Sclera-Matrix. mk Mittelkerne. Zeiss Imm. III. Vergr. $\frac{1300}{1}$.

Fig. 19. Scolopendra cingulata L. A Glaskörper. B Endschläuche mit den Stäbchen von der Fläche gesehen. Vergr. $\frac{1400}{1}$.

Fig. 20. Julus sabulosus L. Querschnitt durch den entkalkten Kopf in der Gegend der Augen (au). Man sieht in der Mitte das Schlundrohr (sch) mit seiner längsfaltigen Chitin- resp. Schleimhaut und den Längs- und Ringmuskeln. Darüber das Gehirn (oberes Schlund- 
ganglion) mit mehrfachen zum Theil sich kreuzenden Faserzügen und den hauptsächlich an der Hirnrinde postirten grossen Ganglienzellen mit Kern und Kernkörperchen. Beiderseits die lobi optici und nach unten, rings um den Schlund, die Komissuren (co) zum unteren kleinen Schlundganglion (uG). m Muskeln mit der sehnigen Platte (s), dr tubulöse (Speichel-?) Drüsen. bl mit Blut gefüllte Hohlräume über dem Gehirn und unter den Augen.

Vergr. $\frac{50}{1}$. Cam. luc.

Fig. 21. Julus sabulosus L. Schnitt durch die unmittelbar aneinander stossenden Einzelaugen nach Entfärbung mit Kalilauge. Der Weichkörper des aggregirten Gesammtauges zeigt sich als eine deutlich doppelblättrige gefaltete aber sehr schmale Lage, welche nach Kalilaugezusatz gelblich erscheint, während die Linsen und die Cuticula überhaupt sich violett färben. pg Stärker pigmentirte oberflächliche Flecke der Cuticula, die man auch zwischen den einzelnen Ocellen findet. no Gemeinsamer an die Augen tretender und sich dort verzweigender nerv. opt. Zeiss F. Vergr. $\frac{160}{1}$.

Fig. 22. Julus sabulosus L. Ein Einzelauge ebendaher mit Zeiss Imm. III angesehen. gl Kerne des Glaskörpers. $r$ grosse (Ganglienzellen?) Kerne in der Retina. Vergr. $\frac{700 .}{1}$

Fig. 23. Lithobius forficatus L. Kopf-Querschnitt. au aggregirte Ocellen. lo lobi optici. he obere Hirn-Hemisphären. bi spongiöses Bindegewebe. $s$ innen gezähneltes Schlundrohr. osm den meisten Tracheaten zukommende Muskeln, womit die obere Schlundwand in die Höhe gezogen, resp. der Schlund erweitert wird. tr Tracheen, $\mathrm{k}$ Mandibeln. km zugehörige Muskeln. Camera lucida Vergr. $\frac{30}{1}$.

Fig. 24. Lithobius forficatus L. Einzelnes mit Oxalsäure entfärbtes Stemma. Man sieht an der Hinterfläche der Linse den aus sehr breiten Pflasterzellen bestehenden Glaskörper und (bei anderer Einstellung) die stäbchenartigen Gebilde der Retina. Ganglienzellen gz (innerhalb der Sclera!) sehr deutlich. Zeiss Imm. III. Vergr. $\frac{300}{1}$.

\section{Tafel VIr.}

Fig. 25. Epeira Schreibersii Dol. Vorderes-Mittel- oder sogenanntes Stirnauge. Vorderste oder frontale Partie eines medianen Tiefenschnittes der Retina und Umgebung nach behutsamer Entfärbung mit Kalilauge mit Zeiss Imm. III. Man beachte die cuticulare Zwischenlamelle (la) vor der Retina, welche sich bei $\checkmark$ mit der Sclera vereinigt, dann die unscheinbaren Kerne am 
Ende der Retinaschläuche (ak). m Augenmuskelquerschnitte.

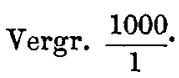

Fig. 26. Epeira Schreibersii Dol. Hinteres Mittel- oder sog. Scheitelauge. Laterale, d. i. gegen die Seitenflanken des Kopfes liegende Partie eines schief von hinten und oben nach vorne und unten gerichteten Schnittes. Links von den typischen stäbchenführenden Schläuchen befindet sich ein besonderer aus Ganglienzellen bestehender Abschnitt (gz) mit einem linsenartig aussehenden Gebilde x. Zeiss Imm. III. Vergr. $\frac{300}{1}$.

Fig. 27. Tegenaria domestica. Zupfpräparat der Scheitelaugen, frisch in Blutserum untersucht. Glaskörperzellen (gl) bis auf eine schwache Körnelung ganz wasserhell, Kerne (a) derselben blass rosa. Ta bläulich grün schimmerndes Tapetum (vrgl. Leydig Histologie pag. 254). ax dünne z. Th. zerbrochene Axenstäbe, gz Ganglienzellen mit blass rosafarbigem Kern. Vergr. $\frac{300}{1}$ Zeiss Imm. L.

Fig. 28. Tegenaria domestica. Partie Retina ebendaher, frisch in Osmiumsäure. Die Axenstäbe erscheinen als spröde, etwas gelblich glänzende Gebilde vom Aussehen eines zarten Chitinhaares. gz Ganglienzellen in einem weitmaschigen Netz schwarzbrauner Pigmentkörner, no Ast des nervus opticus mit seiner chitinösen Scheide (sch). Vergr. $\frac{800}{1}$ (Axenstäbe) $\frac{1200}{1}$. Zeiss. Imm. L.

Fig. 29. Tegenaria domestica. Stück Retina ebendaher frisch im Blut des Thieres. gl Glaskörperzellen. st Stäbchen deutlich rosaviolett gefärbt. ak präbacillärer Kern der Endschläuche (nicht zu verwechseln mit den viel grösseren Glàskörperkernen). Vergr. $\frac{1000}{1}$. Zeiss Imm. L.

Fig. 30. Tegenaria domestica. Krystalloide Plättchen des Tapetums im frischen Zustand mit Zeiss Imm. L. Vergr. $\frac{1200 .}{1}$

Fig. 31. Thomisus sp. Zupfpräparat. Retinale querstreifige (runzelige?) Endschläuche der Scheitelaugen frisch in Osmiumsäure. Glaskörperzellen (gl) scheinbar in kontinuirlichem Zusammenhang mit den Retinalschläuchen. Vergr. $\frac{500}{1}$. 